\title{
Análisis cariotípico de Famatina andina (Phil.) Ravenna (Amaryllidaceae): primer registro de una Hippeastreae sudameri- cana con constricciones secundarias centroméricas
}

\author{
Karyotypic analysis of Famatina andina (Phil.) Ravenna (Amaryllidaceae): first record of South \\ American Hippeastreae with secondary centromeric constrictions
}

\author{
Carlos Baeza ${ }^{1 *} \&$ Jorge Macaya ${ }^{2}$ \\ ${ }^{1}$ Departamento de Botánica, Facultad de Ciencias Naturales y Oceanográficas, Universidad de Concepción, Casilla 160-C, Concepción, Chile. \\ ${ }^{2}$ Cedrem Consultores, Santiago, Chile. \\ *E-mail: cbaeza@udec.cl
}

\begin{abstract}
Bulbs were collected from a population of Famatina andina (Phil.) Ravenna in central Chile. Their chromosomes were analyzed and it was observed that the cariotypic formula and chromosomal number are identical to Famatina cisandina Ravenna. Secondary centromeric constrictions were also observed in chromosomes 1, 2 and 7, and the nucleolus organizing region (NOR) was not observed in the long arm of chromosome 7 . This is the first report of secondary centromeric constrictions in South American Hippeastreae.
\end{abstract}

Amaryllidaceae sensu stricto tiene una distribución mundial y comprende 14 tribus y aproximadamente 70 géneros, en su mayoría geófitos bulbosos, muchos de ellos con una larga historia de cultivo como plantas ornamentales (García et al. 2014). En Chile, se reconocen 23 géneros y cerca de 85 especies (García 2019). Uno de estos géneros es Famatina Ravenna (Hippeastreae), el cual está representado en nuestro país por tres especies. Una de estas especies es $F$. andina (Phil.) Ravenna (Fig. 1A), que crece en Valparaíso, Región Metropolitana y Libertador Bernardo O'Higgins y también en Argentina. Esta especie perenne es poco frecuente, crece en laderas y planicies suaves en los pisos andino y subandino de la Cordillera de los Andes, a veces entre rocas (Teillier et al. 2011). Geófito con bulbo tunicado de forma ovoide, pardo oscuro. Hojas junto a las flores lineales y acintadas de 13 a 15 $\mathrm{mm}$ de ancho, acanaladas, glaucas, con los márgenes amarillorojizos. Escapos florales huecos, de 15 a $30 \mathrm{~cm}$ de longitud, terminados en umbelas de 3 a 6 flores rojas con pedicelos desiguales. Flores tubulosas, rojas, tépalos 6 oblanceolados subiguales, de 3 a $4 \mathrm{~cm}$ de longitud. Estambres 6, exertos, inclinados, unidos a la garganta del tubo, desiguales. Ovario trilocular. Estigma claramente trilobulado. El fruto es una cápsula tricoca con numerosas semillas negras y brillantes (Hoffmann et al. 1998).

García (2019) reconoce tres especies de Famatina para Chile, F. andina, F. cisandina Ravenna y F. maulensis Ravenna (= Miltinea maulensis (Ravenna) Ravenna). Baeza et al. 2017 realizan un estudio citotaxonómico en Famatina cisandina y algunas especies de Rhodophiala C. Presl y concluyen que tanto el número cromosómico, la fórmula cariotípica y los índices cromosómicos $\mathrm{A}_{1}, \mathrm{~A}_{2}, \mathrm{CV}_{\mathrm{CL}}$ y $\mathrm{CV}_{\mathrm{Cl}}$ son muy similares, lo que les permitió concluir que desde una perspectiva citotaxonómica, Famatina cisandina debe ser considerada como una especie de Rhodophiala. De la misma forma, Baeza et al. (2012) estudian una población de Miltinea maulensis (= Famatina maulensis) concluyendo que se trataría de una especie de Phycella Lindl. debido a la enorme similitud en el cariotipo con Ph. australis Ravenna, además de compartir el mismo número cromosómico, prácticamente los mismos valores en los índices cromosómicos indicados anteriormente y la presencia en ambas de una constricción secundaria en el brazo corto del cromosoma cuatro, característica citogenética de Phycella. Estos trabajos han demostrado que el estudio del cariotipo en Amaryllidaceae chilenas es una poderosa 
herramienta para comparar y validar géneros dentro de esta familia.

García et al. (2014) señalan que se conoce el número cromosómico de $F$. cisandina, sin embargo, no se conoce el de F. andina. Por lo tanto, el objetivo de esta comunicación corta es entregar el número cromosómico de esta especie,
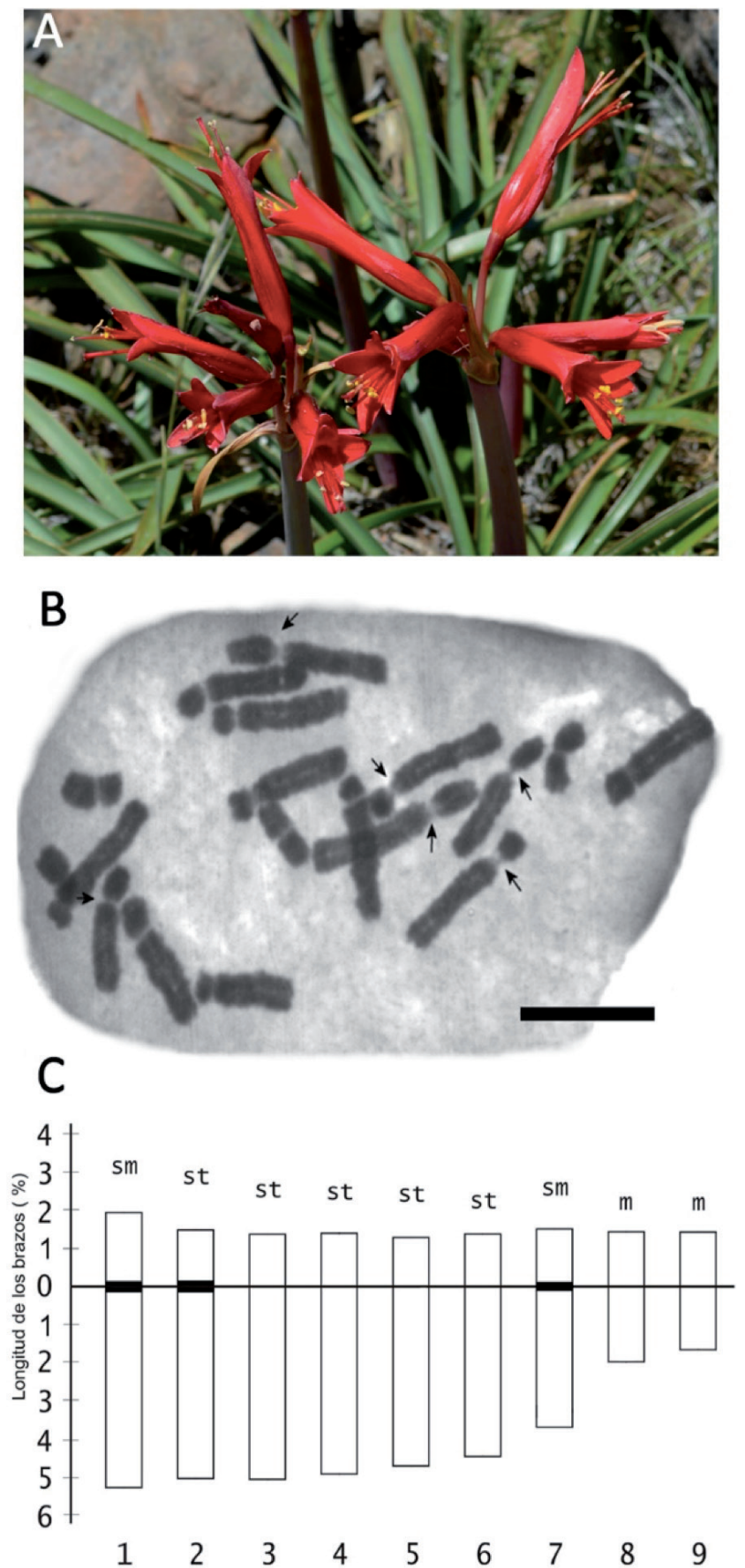

Figura 1. A. Flores de Famatina andina. B. Placa metafásica (las flechas indican las constricciones secundarias centroméricas). C. Idiograma Escala $=10 \mu \mathrm{m}$. / A. Flowers of Famatina andina. B. Metaphase plates (the arrows are indicating the secondary centromeric constrictions). C. Idiogram Scale $=10 \mu \mathrm{m}$. caracterizar sus cromosomas y además reportar sus índices de asimetría correspondientes. García et al. (2019) tratan esta especie como Zephyranthes philippiana Nic.García, pero al parecer este nombre no es correcto y debería usarse otro ( $\mathrm{N}$. García, com. pers.). Por este motivo, consideraremos a esta población como F. andina.

Se analizó una población recolectada en Chile. Región Metropolitana. Cumbre del cerro Cantillana. $2200 \mathrm{~m}$ (335' S - 7057' O). 16-XI-2018. Macaya \& Teillier 1748 (CONC).

Se utilizó tejido meristemático proveniente de ápices radiculares de bulbos cultivados en invernadero; cuando éstos alcanzaron los $8-10 \mathrm{~mm}$, fueron extraídos y se les aplicó un pretratamiento con solución de 8-hidroxiquinolina ( $2 \mathrm{mM}$ ), por $24 \mathrm{~h}$ a $5{ }^{\circ} \mathrm{C}$. Luego, se fijó el material en una solución de etanol absoluto/ácido acético (3:1), por $24 \mathrm{~h}$ a $5{ }^{\circ} \mathrm{C}$. Se procedió a lavar las muestras con agua destilada, a fin de eliminar el fijador, posteriormente fueron sumergidas en $\mathrm{HCl} 0,5 \mathrm{M}$ y colocadas sobre una plancha de secado a $42{ }^{\circ} \mathrm{C}$ durante $16 \mathrm{~min}$. Luego fueron lavadas nuevamente con agua destilada. Sobre un portaobjetos, se procedió a extraer y eliminar la caliptra, se agregó una gota de orceína acética al $1 \%$ para la tinción de los cromosomas y, por último, se hizo el aplastado. Las preparaciones en las que se observaron buenas placas metafásicas fueron almacenadas a $-20{ }^{\circ} \mathrm{C}$ por $24 \mathrm{~h}$ y posteriormente fueron secadas y selladas con Entellán, para finalmente ser fotografiadas y analizadas utilizando un microscopio Zeiss Axioskop, con cámara de video monocromática incluida. Los cromosomas se estudiaron utilizando el software MicroMeasure (Reeves 2001), donde se hicieron las mediciones de los brazos de cada cromosoma del set, respecto al centrómero. El idiograma fue dibujado con el software Corel Draw 8. Se analizaron diez placas metafásicas. Se estimó la asimetría del cariotipo mediante los índices de asimetría intra $A_{1}$, e intercromosomal $A_{2}$ de Romero Zarco (1986) y los coeficientes de variación del índice centromérico $\mathrm{CV}_{\mathrm{CI}}$ y del largo cromosomal CV $\mathrm{CL}_{\mathrm{L}}$ de Peruzzi \& Eroglu (2013). Los cromosomas se clasificaron de acuerdo con Levan et al. (1964, modificado).

La población estudiada presentó $2 \mathrm{n}=2 \mathrm{x}=18$ cromosomas y una fórmula cariotípica haploide compuesta por 2 pares de cromosomas metacéntricos, 2 pares submetacéntricos (el par $n^{\circ} 1$ y 7 con una constricción secundaria centromérica) y 5 pares de cromosomas subtelocéntricos, el par $n^{\circ} 2$ con una constricción secundaria centromérica (Fig. 1B-1C). Los índices de asimetría encontrados fueron $\mathrm{A}_{1} \pm$ D.S. $=0,56 \pm$ 0,$01 ; A_{2} \pm$ D.S. $=0,25 \pm 0,01 ; \mathrm{CV}_{\mathrm{CI}} \pm$ D.S. $=33,5 \pm 2,5$ y CV \pm D.S. $=25,8 \pm 0,6$.

Baeza et al. (2017) caracterizaron los cromosomas de una población de Famatina cisandina concluyendo que se trata de una especie de Rhodophiala, puesto que comparten el mismo número y fórmula cromosómica y los mismos índices de asimetría del cariotipo. Coincidentemente, en este reporte se encontró que $F$. andina presenta las mismas características 
de F. cisandina, en cuanto al número y fórmula cromosómica y a los índices de asimetría analizados, sin embargo, esta población presentó típicamente una constricción secundaria subtelomérica en el brazo largo del cromosoma 7, que corresponde a la región organizadora de nucléolo (NOR) y que se considera como una característica citogenética propia de Rhodophiala (Baeza et al. 2006, 2012). En la población aquí reportada no se observó esta característica en ninguna placa metafásica, no obstante, se observaron constricciones secundarias centrómericas ubicadas en los cromosomas 1, 2 y 7 (Fig. 1B, 1C) en todas las placas estudiadas. Esta condición es primera vez que se señala para algún representante de Hippeastreae sudamericanas, no habiendo registro anterior en otras latitudes.

Por último, con este reporte se terminan de caracterizar citotaxonómicamente los géneros de Amaryllidaceae nativos y endémicos propuestos por Ravenna (2003). Es interesante indicar que la citotaxonomía es una herramienta complementaria potente en este grupo, puesto que los cromosomas de cada uno de estos géneros tienen características propias, son muy estables a nivel poblacional y se pueden diferenciar fácilmente (Baeza \& Schrader 2004, Baeza et al. 2007, 2009a, 2009b, 2012, 2017).

\section{AGRADECIMIENTOS}

Se agradece el apoyo del Departamento de Botánica de la Universidad de Concepción y del Dr. Jaime Espejo.

\section{REFERENCIAS}

Baeza, C., Schrader, O. 2004. Karyotype analysis of Placea amoena Phil. (Amaryllidaceae) by double fluorescence in situ hybridization. Caryologia 57: 209-214.

Baeza, C., Schrader, O., Escobar, I. 2006. Estudio del cariotipo en Rhodophiala aff. advena (Ker-Gawl.) Traub de la VIII Región de Chile. Kurtziana 32: 45-51.

Baeza, M., Ruiz, E., Negritto, M. 2007. El número cromosómico de Phycella australis Ravenna (Amaryllidaceae). Gayana Botánica 64: 117-120.

Baeza, M., Mariangel, C., Ruiz, E., Negritto, M. 2009a. El cariotipo fundamental en Rhodolirium speciosum (Herb.) Ravenna y R. andicola (Poepp.) Ravenna (Amaryllidaceae). Gayana Botánica 66: 99-102.
Baeza, M., Novoa, P., Ruiz, E., Negritto, M. 2009b. El cariotipo fundamental en Traubia modesta (Phil.) Ravenna (Amaryllidaceae). Gayana Botánica 66: 297-300.

Baeza, C., Ruiz, E., Almendras, F., Peñailillo, P. 2012. Estudio comparativo del cariotipo en especies de Miltinea Ravenna, Phycella Lindl. y Rhodophiala C. Presl (Amaryllidaceae) de Chile. Revista de la Facultad de Ciencias Agrarias Uncuyo 44: 197-209.

Baeza, C., García, N., Herrera, F., Ruiz, E., Rosas, M. 2017. Chromosomal characterization of Rhodolirium laetum (Phil.) Ravenna (Amaryllidaceae) through karyotyping and in-situ hybridization of ribosomal DNA. Gayana Botánica 74: 240-244.

Baeza, C., Herrera, F., Ruiz, E., Peñailillo, P., Novoa, P., Rosas, M. 2017. ¿Es Famatina cisandina Ravenna (Amaryllidaceae) una especie válida desde una perspectiva citológica? Chloris Chilensis 20: 1. URL: http://www.chlorischile.cl

García, N. 2019. Amaryllidaceae. 24-30 pp. En: Rodríguez, R., Marticorena, A. (Eds.). Catálogo de las Plantas Vasculares de Chile. Editorial Universidad de Concepción, Chile.

García, N., Meerow, A., Soltis, D., Soltis, P. 2014. Testing deep reticulate evolution in Amaryllidaceae tribe Hippeastreae (Asparagales) with ITS and chloroplast sequence data. Systematic Botany 39: 75-89.

García, N., Meerow, A., Arroyo-Leuenberger, S., Oliveira, R., Dutilh, J., Soltis, P., Judd W. 2019. Generic classification of Amaryllidaceae tribe Hippeastreae. Taxon 68: 481-498.

Hoffmann, A., Liberona, F., Muñoz, M., Watson, J. 1998. Plantas altoandinas en la flora silvestre de Chile. Ediciones Fundación Claudio Gay, Santiago, Chile. 281 pp.

Levan, A., Fredga, K., Sandber, A. 1964. Nomenclature for centromeric position on chromosomes. Hereditas 52: 201-220.

Peruzzi, L., Eroglu, H. 2013. Karyotype asymmetry: again, how to measure and what to measure? Comparative Cytogenetics 7: 1-9.

Ravenna, P. 2003. Elucidation and systematics of the Chilean genera of Amaryllidaceae. Botanica Australis 2: 1-21.

Reeves, A. 2001. MicroMeasure: a new computer program for the collection and analysis of cytogenetic data. Genome 44: 239-443.

Romero Zarco, C. 1986. A new method for estimating karyotype asymmetry. Taxon 35: 526-530.

Teillier, S., Marticorena, A., Niemeyer, H. 2011. Flora andina de Santiago. Santiago, Chile. 478 pp.

Received: 25.11.2019

Accepted: 03.03.2020 